\title{
一种可视化检测硫化氢的苯并噻唑类荧光增强型探针
}

\author{
范方禄* 靖金球＼cjkstart陈雪梅 \\ (湖北理工学院化学与材料工程学院 黄石 435003)
}

\begin{abstract}
摘要 以叠氮基为识别基团，合成和表征了一种苯并噻唑类荧光增强型硫化氢探针(FL), 并研究了它的光谱性质. 实 验结果表明, FL 对硫化氢有较高的选择性和灵敏度, 且响应快速, 抗干扰能力强, 细胞毒性低, 它的检出限度为 $8.78 \times$ $10^{-7} \mathrm{~mol} \cdot \mathrm{L}^{-1}$, 在 HeLa 活细胞和各种水体样品中对硫化氢的检测表明 FL 具有潜在的应用价值.
\end{abstract}

关键词 硫化氢; 荧光探针; 叠氮基; 可视化

\section{A Benzothiazole-Derived Fluorescence Enhancement Probe for Visual Detection of $\mathrm{H}_{2} \mathrm{~S}$}

\author{
Fan, Fanglu* Jing, Jinqiu Chen, Xuemei \\ (School of Chemical and Materials Engineering, Hubei Polytechnic University, Huangshi 435003)
}

\begin{abstract}
A benzothiazole-derived fluorescence enhancement probe (FL) for the detection of $\mathrm{H}_{2} \mathrm{~S}$ was synthesized and characterized by employing azide group as the recognition unit, and its spectral properties had been researched. The results showed that FL exhibited relatively good sensitivity and selectivity to $\mathrm{H}_{2} \mathrm{~S}$ as well as fast response, it also had strong anti-interference ability and low cytotoxicity, the detection limit of $\mathrm{H}_{2} \mathrm{~S}$ was estimated to be $8.78 \times 10^{-7} \mathrm{~mol} \cdot \mathrm{L}^{-1}$, and the detection of $\mathrm{H}_{2} \mathrm{~S}$ in living HeLa cells and various water samples indicated that it had potential application value.
\end{abstract}

Keywords $\mathrm{H}_{2} \mathrm{~S}$; fluorescent probe; azide group; visualization

硫化氢 $\left(\mathrm{H}_{2} \mathrm{~S}\right)$ 是一种无色、易燃、易溶于水的酸性气 体, 它广泛存在于自然界中的原油、天然气、火山气和 温泉中, 是继一氧化氮(NO)和一氧化碳 $(\mathrm{CO})$ 之后的第 三种气体信号分子 ${ }^{[1]} . \mathrm{H}_{2} \mathrm{~S}$ 一直被认为是一种带有臭蛋 气味的对环境有害的气体, 它主要由有机物腐败而产 生, 估计全世界每年进入大气的量约 1 亿吨，人为产生 的硫化氢每年约 300 万吨, 主要来源是牛皮纸浆厂、炼 焦厂炼油厂和人造丝厂. 除了对大气的污染外, 含 $\mathrm{H}_{2} \mathrm{~S}$ 的水会发臭, 对混凝土和金属都也有侵蚀破坏作用. 作 为生活饮用水或工业用水的水源, 水中的 $\mathrm{H}_{2} \mathrm{~S}$ 应完全去 除. 此外, 也有更多的研究发现 $\mathrm{H}_{2} \mathrm{~S}$ 在许多生理过程中 诸如调制血压、传递调控神经信号等方面扮演着重要角

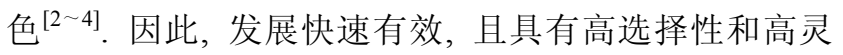
敏度的检测 $\mathrm{H}_{2} \mathrm{~S}$ 的方法已成为当前研究的热点领域之 一. 近年来, 直观、操作简单、灵敏度高的小分子荧光 探针的出现引起了广泛关注. 基于 $\mathrm{H}_{2} \mathrm{~S}$ 的的双亲核性、 还原性、强的 $\mathrm{Cu}-\mathrm{S}$ 键合能力和能与不饱和双键加成等
化学特性, 已经报道了多种 $\mathrm{H}_{2} \mathrm{~S}$ 荧光探针 ${ }^{[5 \sim 12]}$. 目前, Chang 和 Wang 等 ${ }^{[13,14]}$ 报道的将叠氮基还原为氨基的苂 光增强型的 $\mathrm{H}_{2} \mathrm{~S}$ 荧光探针被广泛接受和应用. 这类探针 是通过改变含有不同含叠氮基的荧光团来设计的，已报 道的苂光团有萗酰亚胺、苯并氧杂噁二唑、花菁、香豆 素、甲酚紫、萠等 ${ }^{[15 ~ 20]}$. 然而, 开发这类能在细胞、组 织、活体或者污染水体中对 $\mathrm{H}_{2} \mathrm{~S}$ 进行实时传感，且具有 高灵敏度和可视化成像的探针仍是一大挑战. 平面型的 苯并噻唑类化合物大多具有强烈的荧光和较高的荧光 量子产率等突出的光物理和光化学性能, 它是制作荧光 增白剂、苂光染料的重要原料, 在高灵敏度显色剂的合 成中也具有重要的地位 ${ }^{[21,22]}$. 鉴于此, 本文以苯并噻唑 为荧光团, 基于 $\mathrm{H}_{2} \mathrm{~S}$ 对叠氮基较好的还原能力, 设计合 成了一种可用于检测 $\mathrm{H}_{2} \mathrm{~S}$ 的苂光探针 2-(4-叠氮基苯基) 苯并噻唑(FL). 该探针分子能选择性地对 $\mathrm{H}_{2} \mathrm{~S}$ 表现出苂 光增强响应, 在 HeLa 活细胞和各种水体样品中对硫化 氢的检测表明 FL 具有潜在的应用前景.

*E-mail: flfan2014@163.com

Received April 9, 2014; revised May 5, 2014; published online June 3, 2014.

Project supported by the National Natural Science Foundation of China (No. 51173060).

国家自然科学基金(No. 51173060)资助项目. 


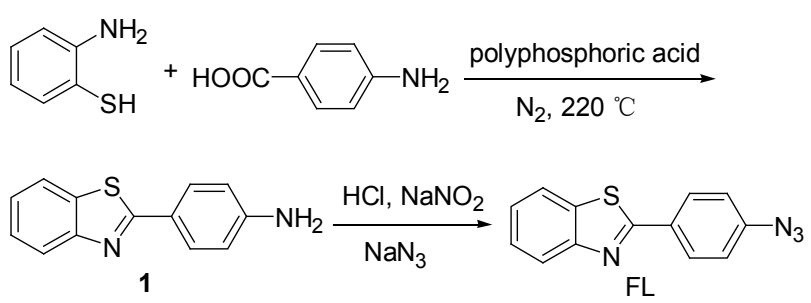

Scheme 1

\section{1 结果与讨论}

\section{1 晶体结构}

以对氨基苯甲酸和邻氨基苯硫酚为起始原料, 通过 两步反应合成了荧光探针 FL(图 1), 将其在乙酸乙酯中 溶解, 室温静置 $3 \mathrm{~d}$ 后可得到适合 $X$ 射线单晶测试的淡 黄色块状晶体. 苂光探针 $\mathrm{FL}$ 分子式为 $\mathrm{C}_{13} \mathrm{H}_{8} \mathrm{~N}_{4} \mathrm{~S}$, 分子 量为 252.30. X 射线单晶衍射测试表明该化合物属于斜 方晶系, 空间群为 $P b c a$, 晶胞参数 $a=14.501(2) \AA, b=$ 5.8589(10) $\AA, c=27.096(5) \AA, \alpha=90^{\circ}, \beta=90^{\circ}, \gamma=90^{\circ}$, $V=2.3021(7) \mathrm{nm}^{3}, D_{\mathrm{c}}=1.456 \mathrm{~g} \cdot \mathrm{cm}^{-3}, Z=8, F(000)=$ $1040, R_{1}=0.0461, w R_{2}=0.1173[I>2 \sigma(I)]$. 晶体结构数 据存于英国剑桥数据中心, CCDC 号为 978109 .

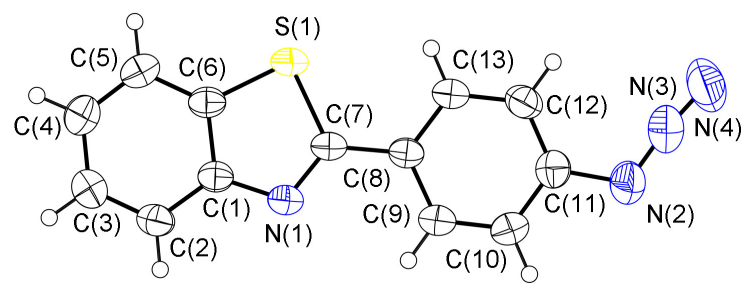

图 1 苂光探针 FL 的晶体结构

Figure 1 The crystal structure of fluorescent probe FL

表 $1 \mathrm{FL}$ 的部分键长 $(\AA)$ 和键角 $\left({ }^{\circ}\right)$ 数据

Table 1 Selected bond lengths $(\AA)$ and bon angles $\left(^{\circ}\right)$ of FL

\begin{tabular}{llll}
\hline $\mathrm{C}(11)-\mathrm{N}(2)$ & $1.425(3)$ & $\mathrm{C}(7)-\mathrm{N}(1)$ & $1.300(2)$ \\
$\mathrm{N}(2)-\mathrm{N}(3)$ & $1.240(3)$ & $\mathrm{N}(3)-\mathrm{N}(4)$ & $1.122(3)$ \\
$\mathrm{C}(1)-\mathrm{N}(1)$ & $1.387(3)$ & $\mathrm{C}(6)-\mathrm{S}(1)$ & $1.728(2)$ \\
$\mathrm{N}(4)-\mathrm{N}(3)-\mathrm{N}(2)$ & $173.0(3)$ & $\mathrm{C}(6)-\mathrm{S}(1)-\mathrm{C}(7)$ & $89.11(9)$ \\
$\mathrm{N}(1)-\mathrm{C}(7)-\mathrm{C}(8)$ & $124.12(17)$ & $\mathrm{C}(8)-\mathrm{C}(7)-\mathrm{S}(1)$ & $120.09(13)$ \\
\hline
\end{tabular}

在荧光探针 FL 的晶体结构中(图 1), 苯并噻唑环和 与之相连的苯环并不共平面, 它们之间的二面角为 $5.01^{\circ}$, 与苯环相连的叠氮基的 $\mathrm{C}-\mathrm{N}$ 键长 $[\mathrm{C}(11)-\mathrm{N}(2)$, $0.1425(3) \mathrm{nm}]$ 稍长于苯并噻唑环的 $\mathrm{C}-\mathrm{N}$ 键长 $[\mathrm{C}(7)$ $\mathrm{N}(1), 0.1300(2) \mathrm{nm}$ ] (表 1). 叠氮基的两个 $\mathrm{N}-\mathrm{N}$ 键长并 不相等 $[\mathrm{N}(2)-\mathrm{N}(3), 0.1240(3) \mathrm{nm} ; \mathrm{N}(3)-\mathrm{N}(4), 0.1122(3)$ $\mathrm{nm}]$, 三个氮原子间的夹角为 $173.0(3)^{\circ}$, 为非直线型结 构.

\section{2 荧光探针 FL 的光谱性质}

在乙醇溶液中, FL 和中间体 $\mathbf{1}$ 的最大激发波长 $\left(\lambda_{\mathrm{ex}}\right)$ 分别为 325 和 $350 \mathrm{~nm}$, 最大发射波长 $\left(\lambda_{\mathrm{em}}\right)$ 为分别为 415 和 $421 \mathrm{~nm}$ ，但相同浓度的探针 FL $\left(10 \mu \mathrm{mol} \cdot \mathrm{L}^{-1}\right)$ 的荧光 强度相比中间体 1 明显要弱很多(图 2), 它们的苂光量子 产率分别为 $0.0078,0.4026$. 这是由于 2 -芳基苯并噻唑 为非对称的刚性平面结构, 内含大的共轭体系, 具有 强吸电子能力, 当探针 FL 的吸电子基团叠氮基转化 为具有强的供电子能力的氨基, 分子内电荷转移 (ICT)效应增强，使得中间体 1 具有较强的荧光.

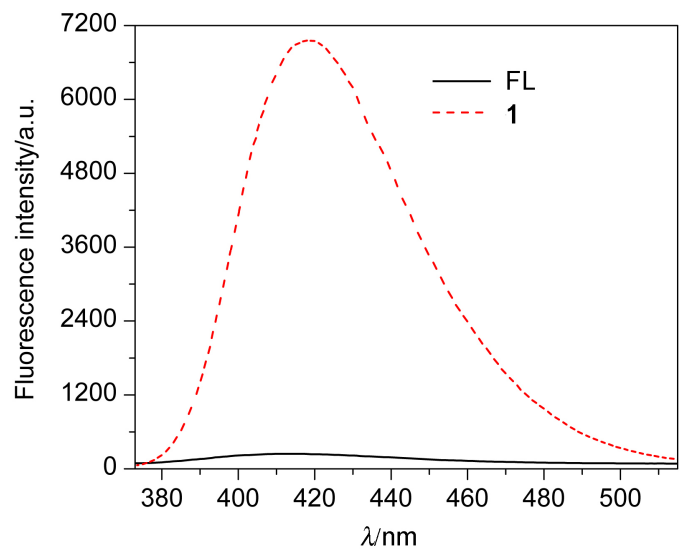

图 2 探针 $\mathrm{FL}\left(10 \mu \mathrm{mol} \cdot \mathrm{L}^{-1}\right)$ 和中间体 $\mathbf{1}\left(10 \mu \mathrm{mol} \cdot \mathrm{L}^{-1}\right)$ 的荧光 光谱

Figure 2 The fluorescence spectra of probe FL $\left(10 \mu \mathrm{mol} \cdot \mathrm{L}^{-1}\right)$ and intermediate $\mathbf{1}\left(10 \mu \mathrm{mol} \cdot \mathrm{L}^{-1}\right)$

在 $\mathrm{CH}_{3} \mathrm{CH}_{2} \mathrm{OH} / \mathrm{Tris}-\mathrm{HCl}(V / V=1 / 1, \mathrm{pH}=7.4)$ 缓冲体 系中, 我们研究了 FL $\left(10 \mu \mathrm{mol} \cdot \mathrm{L}^{-1}\right)$ 对不同物质 $(100$ $\left.\mu \mathrm{mol} \cdot \mathrm{L}^{-1}\right)$ 的荧光响应. 如图 3 所示, 在 $\mathrm{FL}$ 中加入 $\mathrm{F}^{-}$, $\mathrm{Cl}^{-}, \mathrm{Br}^{-}, \mathrm{I}^{-}, \mathrm{SCN}^{-}, \mathrm{HCO}_{3}^{-}, \mathrm{CO}_{3}^{2-}, \mathrm{PO}_{4}^{3-}, \mathrm{SO}_{4}^{2-}$ 等各 种阴离子, 其荧光强度几乎没有什么变化, 活性氧物种 $\left(\mathrm{H}_{2} \mathrm{O}_{2}, \mathrm{ClO}^{-}\right)$和生物硫醇 $(\mathrm{Cys}, \mathrm{GSH})$ 也没有明显改变它 的荧光强度, 而加入 $\mathrm{NaHS}$ (一种常用的 $\mathrm{H}_{2} \mathrm{~S}$ 供体)之后, $F L$ 的荧光显著增强. 因此, FL 可以作为一种检测 $\mathrm{H}_{2} \mathrm{~S}$ 的 有较高选择性的荧光增强型探针. 为了验证 FL 是否具 有专一的选择性和较强的抗干扰能力, 我们选择了细胞 及生物体中常见的各种阴阳离子 $\left(\mathrm{Cl}^{-}, \mathrm{HCO}_{3}^{-}, \mathrm{CO}_{3}^{2-}\right.$, $\left.\mathrm{H}_{2} \mathrm{PO}_{4}^{-}, \mathrm{SO}_{4}^{2-}, \mathrm{K}^{+}, \mathrm{Ca}^{2+}, \mathrm{Na}^{+}, \mathrm{Mg}^{2+}, \mathrm{Cu}^{2+}, \mathrm{Zn}^{2+}\right)$ 、活性 氧物种 $\left(\mathrm{H}_{2} \mathrm{O}_{2}, \mathrm{ClO}^{-}\right)$和生物硫醇 (Cys, GSH) 作为测试的 干扰物. 从图 4 可以看出, 当 $\mathrm{NaHS}$ 的浓度为 $10 \mu \mathrm{mol} / \mathrm{L}$, 加入 100 倍量的各种干扰物后，探针 FL 仍可以有效的 对 $\mathrm{H}_{2} \mathrm{~S}$ 进行检测，干扰物的存在几乎没有改变 FL 的苂 光, 这也进一步证实了 $\mathrm{FL}$ 对 $\mathrm{H}_{2} \mathrm{~S}$ 的良好选择性. 


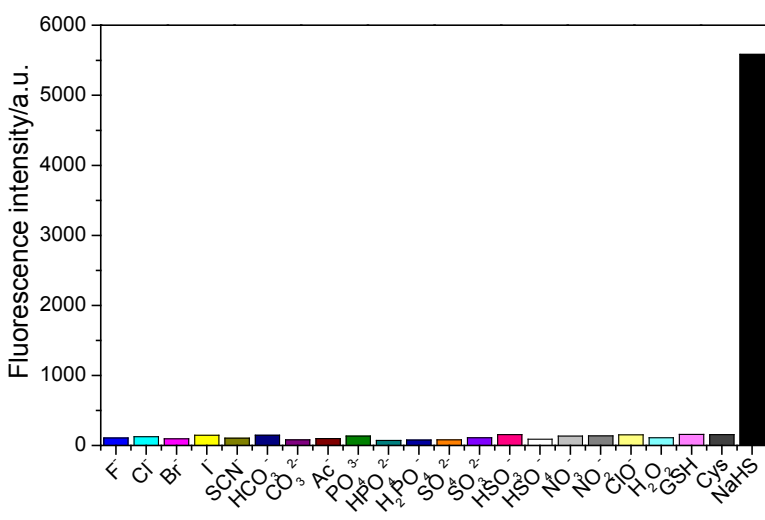

图 3 探针 $\mathrm{FL}\left(10 \mu \mathrm{mol} \cdot \mathrm{L}^{-1}\right)$ 对不同物质 $\left(100 \mu \mathrm{mol} \cdot \mathrm{L}^{-1}\right)$ 的苂光 响应

Figure 3 The fluorescent response of probe FL $\left(10 \mu \mathrm{mol} \cdot \mathrm{L}^{-1}\right)$ to different species $\left(100 \mu \mathrm{mol} \cdot \mathrm{L}^{-1}\right)$

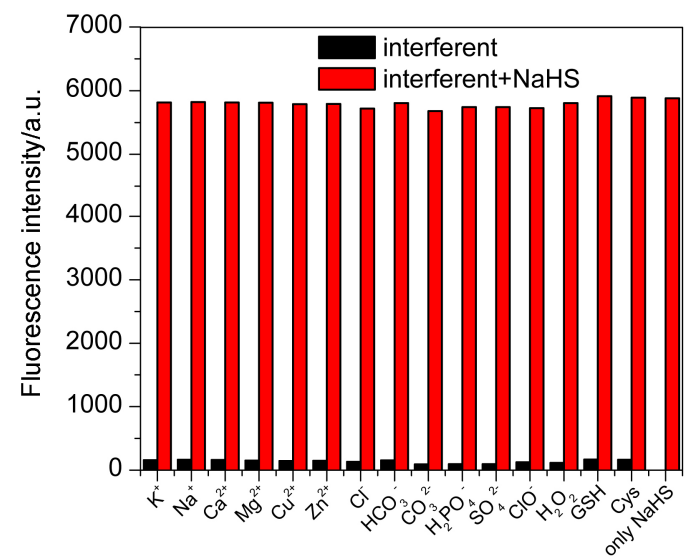

图 4 在各种干扰物 $(1000 \mu \mathrm{mol} / \mathrm{L})$ 的存在下 $\mathrm{NaHS}$ 对探针 $\mathrm{FL}$ $(10 \mu \mathrm{mol} / \mathrm{L})$ 苂光的影响

Figure 4 Effect of NaHS on probe FL $(10 \mu \mathrm{mol} / \mathrm{L})$ in the presence of different interferents $(1000 \mu \mathrm{mol} / \mathrm{L})$

荧光滴定实验证实探针 $\mathrm{FL}$ 对 $\mathrm{H}_{2} \mathrm{~S}$ 响应灵敏(图 5). FL 自身的荧光较弱，向其中逐渐加入 NaHS 后，它在 $421 \mathrm{~nm}$ 处的发射峰荧光强度逐渐增大, 表明含有叠氮 基的探针被 $\mathrm{H}_{2} \mathrm{~S}$ 还原为中间体 $\mathbf{1}$, 此时反应液的质谱也 证实了这一结果[产物中间体 $\mathbf{1}$ 的 $\mathrm{m} / \mathrm{z}$ 为 $226.17\left(\mathrm{M}^{+}\right)$]. 当 $\mathrm{NaHS}$ 浓度增大到 $50 \mu \mathrm{mol} \cdot \mathrm{L}^{-1}$ 时, $\mathrm{FL}$ 的苂光强度基 本稳定; 继续将其浓度增大到 $100 \mu \mathrm{mol} \cdot \mathrm{L}^{-1}$ 时, FL 的苂 光强度也没有发生太大的变化, 说明反应体系的荧光强 度已经达到饱和. 和没有加入 NaHS 的情况相比, 此时 探针的荧光强度大约增强了 50 倍. 在实验中还发现, FL 的荧光强度与 NaHS $\left(0 \sim 6.5 \mu \mathrm{mol} \cdot \mathrm{L}^{-1}\right)$ 有较好的线性关 系(线性相关系数: $R^{2}=0.9931$ ), 表明 FL 可以用来定量 检测 $\mathrm{H}_{2} \mathrm{~S}$ 的浓度(图 6). 根据文献的计算方法 ${ }^{[23]}$, FL 对 $\mathrm{H}_{2} \mathrm{~S}$ 的检出限度为 $8.78 \times 10^{-7} \mathrm{~mol} \cdot \mathrm{L}^{-1}$. 此外, FL 对 $\mathrm{NaHS}$ 响应也较快(图 7). 向 FL $\left(10 \mu \mathrm{mol} \cdot \mathrm{L}^{-1}\right)$ 中加入 5 equiv. 的 NaHS, $5 \mathrm{~min}$ 后反应体系的苂光强度基本恒定,

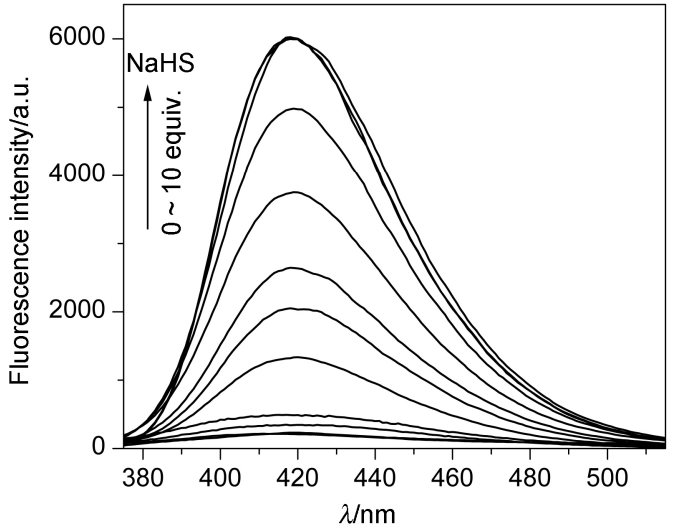

图 $5 \mathrm{FL}\left(10 \mu \mathrm{mol} \cdot \mathrm{L}^{-1}\right)$ 在不同浓度的 $\mathrm{NaHS}$ 溶液 $(0 \sim 100$ $\left.\mu \mathrm{mol} \cdot \mathrm{L}^{-1}\right)$ 中的苂光光谱

Figure 5 The fluorescence spectra of FL $\left(10 \mu \mathrm{mol} \cdot \mathrm{L}^{-1}\right)$ in the presence of NaHS $\left(0 \sim 100 \mu \mathrm{mol} \cdot \mathrm{L}^{-1}\right)$

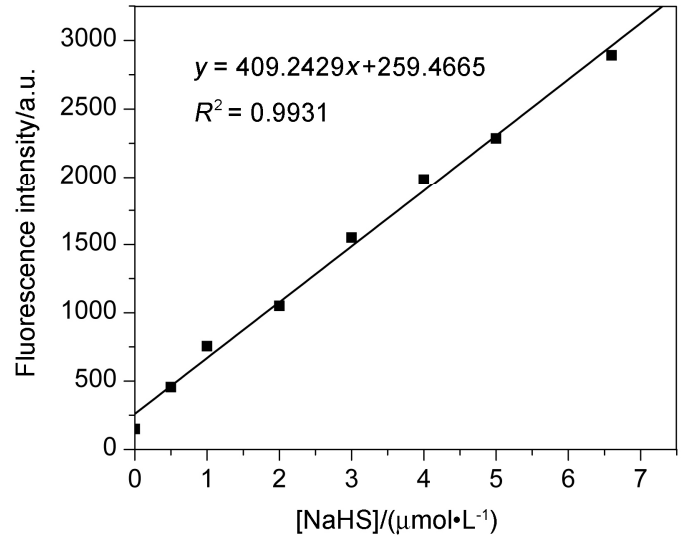

图 $6 \mathrm{FL}$ 的荧光强度随 $\mathrm{NaHS}$ 浓度 $\left(0 \sim 6.5 \mu \mathrm{mol} \cdot \mathrm{L}^{-1}\right)$ 变化的线 性关系图

Figure 6 Fluorescence intensity of FL as a function of concentration of $\mathrm{NaHS}\left(0 \sim 6.5 \mu \mathrm{mol} \cdot \mathrm{L}^{-1}\right)$

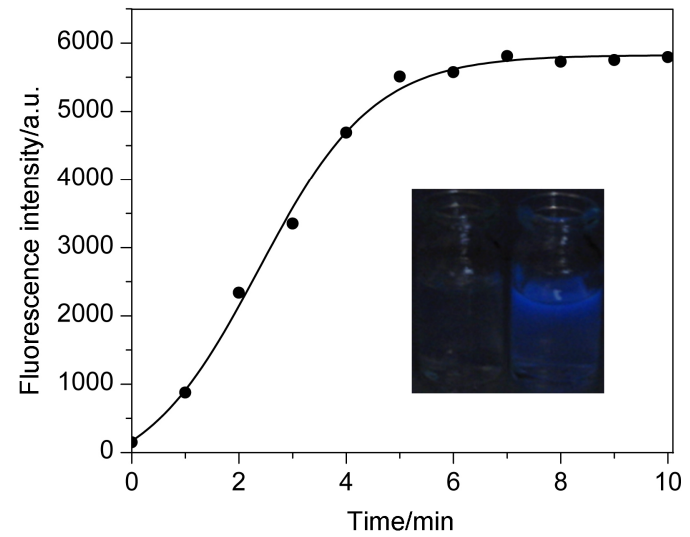

图 7 探针 $\mathrm{FL}\left(10 \mu \mathrm{mol} \cdot \mathrm{L}^{-1}\right)$ 在加入 5 equiv. 的 $\mathrm{NaHS}$ 后荧光光 谱随时间的变化.

Figure 7 Time-dependent fluorescence spectral changes of FL $\left(10 \mu \mathrm{mol} \cdot \mathrm{L}^{-1}\right)$ in the presence of $\mathrm{NaSH}$ ( 5 equiv.)

The inset shows the fluorescence color of FL before (left) and after (right) addition of NaHS for $5 \mathrm{~min}$ (UV lamp, $365 \mathrm{~nm}$ )

说明此时 NaHS 对 FL 的还原反应达到了平衡状态. 将 
该种反应液用波长为 $365 \mathrm{~nm}$ 的紫外灯照射, 溶液的颜 色由没有加入 NaHS 前的极其微弱的浅蓝色变成了亮蓝 色, 这种明显的颜色和荧光强度变化, 表明 FL 对 $\mathrm{H}_{2} \mathrm{~S}$ 有良好的识别作用(图 7 插图).

我们还考察了 $\mathrm{pH}$ 值对探针 $\mathrm{FL}$ 识别 $\mathrm{H}_{2} \mathrm{~S}$ 的影响. 由 图 8 可知, 在未加 $\mathrm{NaHS}$ 前, FL 在 $\mathrm{pH} 2 \sim 12$ 的范围内无 明显荧光响应. 当入 $\mathrm{NaHS}$ 后, $\mathrm{FL}$ 在 $\mathrm{pH} 7 \sim 12$ 范围内产 生强而稳定的荧光响应信号, 这表明 FL 可以在较宽的 $\mathrm{pH}$ 范围内对 $\mathrm{H}_{2} \mathrm{~S}$ 进行检测.

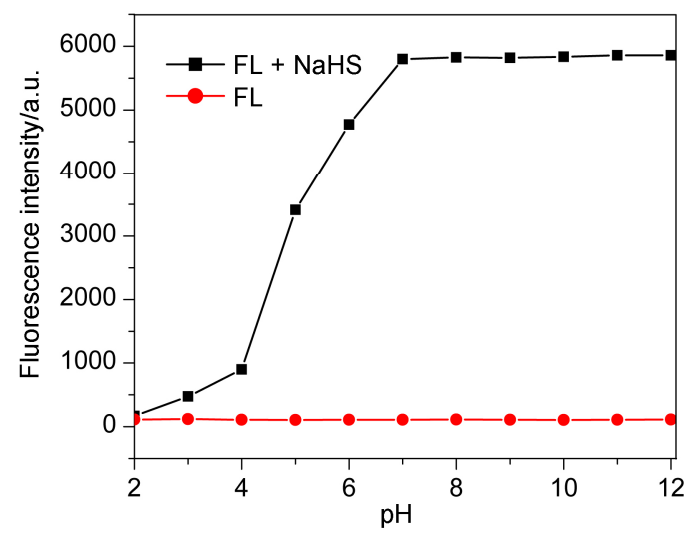

图 8 在 $\mathrm{NaHS}(100 \mu \mathrm{mol} / \mathrm{L})$ 存在或不存在的条件下, FL (10 $\mu \mathrm{mol} / \mathrm{L}$ ) 在不同 $\mathrm{pH}$ 值时荧光强度的变化(FL 的荧光强度是在加 入 $\mathrm{NaSH} 5 \mathrm{~min}$ 后测试的)

Figure 8 Changes in fluorescence intensity of FL $(10 \mu \mathrm{mol} \cdot$ $\left.\mathrm{L}^{-1}\right)$ measured in the absence and presence of NaHS (100 $\mu \mathrm{mol} / \mathrm{L}$ ) as a function of $\mathrm{pH}$ (The fluorescence intensity of FL upon addition of NaHS was measured after $5 \mathrm{~min}$ )

\section{3 探针 $\mathrm{FL}$ 对细胞内 $\mathrm{H}_{2} \mathrm{~S}$ 的造影及其细胞毒性}

将 $5 \mu \mathrm{mol} \cdot \mathrm{L}^{-1}$ 探针 $\mathrm{FL}$ 的乙醇溶液加入到预先培养 好的 $\mathrm{HeLa}$ 细胞中, 在培养基中于 $37^{\circ} \mathrm{C}$ 培养 $30 \mathrm{~min}$, 用 PBS 缓冲液洗涤后进行细胞造影. 荧光图像显示在 $\mathrm{HeLa}$ 活细胞中并未见明显的荧光分布, 再向上述含 $\mathrm{FL}$ 的细胞培养液中加入 $\mathrm{NaHS}\left(100 \mu \mathrm{mol} \cdot \mathrm{L}^{-1}\right)$ 并在 $37{ }^{\circ} \mathrm{C}$ 的 条件下捊化 $30 \mathrm{~min}$, 可观察到 HeLa 活细胞呈现出明亮 的蓝色苂光, 证实了 $\mathrm{FL}$ 能在细胞内对 $\mathrm{H}_{2} \mathrm{~S}$ 进行造影. 与 之对应的亮场图像没有发现细胞调亡现象, 这表明探针 FL 具有良好的细胞相容性和细胞膜透过能力, 可能在 检测生物样品中的 $\mathrm{H}_{2} \mathrm{~S}$ 方面有潜在的应用.

探针的细胞毒性大小是评判它是否适合生物应用 的一个重要方面. 我们用 MTT 法在 HeLa 细胞中对探针 FL 和中间体 1 进行了细胞毒性的检测. 测试时选用与细 胞造影实验相同的探针浓度 $\left(5 \mu \mathrm{mol} \cdot \mathrm{L}^{-1}\right)$, 实验结果表 明, 探针 $\mathrm{FL}$ 和中间体 1 ( $\mathrm{FL}$ 被 $\mathrm{H}_{2} \mathrm{~S}$ 还原的产物)在细胞 中培养 $12 \mathrm{~h}$ 后, 细胞的存活率分别为 $97 \%$ 和 $96 \%$, 这说 明它们对细胞产生毒害作用很小. 此外, 继续使探针 FL 和中间体 1 在 HeLa 细胞中培养 $24 \mathrm{~h}$ 后, 发现细胞的存
活率仍达到 95\%左右，因此 FL 和中间体 1 的细胞毒性 较小, 适合长时间地在活细胞中实现对 $\mathrm{H}_{2} \mathrm{~S}$ 的检测.

\section{4 探针 FL 对各种水体样品中硫化氢的检测}

为了考察探针 FL 的实际应用价值，我们取自来水、 湖水和工业废水来进行硫化氢的恢复性试验. 从表 2 的 结果可以看出，自来水和湖水中由于硫化氢的浓度较 低，探针 FL 检测不出，而测得工业废水样品中硫化氢 浓度为 $3.52 \mu \mathrm{mol} \cdot \mathrm{L}^{-1}$. 此外, 向自来水和湖水加入外源 硫化氢，测试结果的相对标准偏差分别为 $1.33 \%$ 和 $3.57 \%$ ，而向工业废水中加入外源硫化氢，检测的恢复 率可达 $93.5 \%$ ，这表明探针 FL 用于检测这些水体样品 中硫化氢具有较好的选择性和灵敏度，且具有一定的实 用价值.

表 2 检测各种水体样品中硫化氢的恢复试验

Table 2 Recovery study of the detection of $\mathrm{H}_{2} \mathrm{~S}$ in various water samples

\begin{tabular}{ccccc}
\hline 水体样品 & $\begin{array}{c}\mathrm{H}_{2} \mathrm{~S} \text { 加入 } \\
\left(\mu \mathrm{mol} \cdot \mathrm{L}^{-1}\right)\end{array}$ & $\begin{array}{c}\mathrm{H}_{2} \mathrm{~S} \text { 测试 } \\
\left(\mu \mathrm{mol} \cdot \mathrm{L}^{-1}\right)\end{array}$ & 恢复率 $/ \%$ & $\begin{array}{c}\text { 相对标准 } \\
\text { 偏差 } / \%\end{array}$ \\
\hline 自来水 & 0 & 不能检测 & - & - \\
& 2.00 & 1.95 & 97.5 & 1.33 \\
& 4.00 & 3.94 & 98.5 & \\
湖水 & 6.00 & 6.06 & 100.1 & - \\
& 0 & 不能检测 & - & - \\
工业废水 & 2.00 & 1.91 & 95.5 & 3.57 \\
& 6.00 & 4.06 & 101.5 & \\
\hline
\end{tabular}

\section{2 结论}

本文合成了一种含有叠氮基识别基团的苯并噻唑 类荧光增强型硫化氢探针(FL), 它能较快地识别 $\mathrm{H}_{2} \mathrm{~S}$, 而且具有较高的选择性和灵敏度, 其检出限度为 $8.78 \times$ $10^{-7} \mathrm{~mol} \cdot \mathrm{L}^{-1}$. 此外, 在 HeLa 活细胞和各种水体样品中 对硫化氢的检测表明 FL 可能具有在生物和环境方面潜 在的应用价值.

\section{3 实验部分}

\section{1 主要仪器与试剂}

实验所用试剂均为市售分析纯. PHS-3C 型 $\mathrm{pH}$ 计(上 海仪电科学仪器股份有限公司); Analytik jena Specord 210 型紫外可见分光光度计(德国耶拿分析仪器公司); XT4-100X 显微熔点测定仪(上海申光仪器仪表有限公 司); Varian Mercury $400 \mathrm{MHz}$ 核磁共振仪(TMS 作内标, 美国 Varian 公司); Applied Biosystems API 2000 LC/MS/ MS 电喷雾质谱仪(美国应用生物系统公司); Brucker 
AXS SMART 2000 CCD 单晶衍射仪(德国 Brucker 公司); VARIAN-CARY 荧光光谱仪(美国 Varian 公司); Leica DMI 3000B 倒置荧光显微镜(德国徕卡仪器有限公司); SpectraMax Plus384 型酶标仪(美国分子仪器公司).

\section{2 合成}

\subsubsection{2-(4-氨基苯基)苯并噻唑(1)的合成}

中间体 1 的合成参照文献[24]的方法并稍作改进. 以 多聚磷酸为溶剂, 将邻氨基苯硫酚 $(1.25 \mathrm{~g}, 10 \mathrm{mmol})$ 和 对氨基苯甲酸 $(1.37 \mathrm{~g}, 10 \mathrm{mmol})$ 混合在一起, 在氮气的 保护下于 $220{ }^{\circ} \mathrm{C}$ 反应 $3 \mathrm{~h}$. 当反应液稍冷后, 将其倒入 冰水中, 再向其中缓慢加入 $\mathrm{Na}_{2} \mathrm{CO}_{3}$ 并不断搅拌, 调节 溶液的 $\mathrm{pH}$ 为 7 , 过滤, 所得残渣经柱层析分离 $[V($ 乙酸 乙酯)： $V$ (石油醚 $)=1: 5$ ]得到淡黄色的晶状粉末 2.03 g, 产率 90\%. m.p. 156.2 157.8 ${ }^{\circ} \mathrm{C}$; ${ }^{1} \mathrm{H}$ NMR $(400 \mathrm{MHz}$, $\left.\mathrm{CDCl}_{3}\right) \delta: 4.02(\mathrm{~s}, 2 \mathrm{H}), 6.72(\mathrm{~d}, J=7.2 \mathrm{~Hz}, 2 \mathrm{H}), 7.33(\mathrm{t}$, $J=7.6 \mathrm{~Hz}, 1 \mathrm{H}), 7.45$ (t, $J=7.6 \mathrm{~Hz}, 1 \mathrm{H}), 7.86(\mathrm{~d}, J=8.8$ $\mathrm{Hz}, 2 \mathrm{H}), 7.91(\mathrm{~d}, J=7.2 \mathrm{~Hz}, 1 \mathrm{H}), 8.00(\mathrm{~d}, J=8.8 \mathrm{~Hz}, 2 \mathrm{H})$; ${ }^{13} \mathrm{C} \mathrm{NMR}\left(100 \mathrm{MHz}, \mathrm{CDCl}_{3}\right) \delta: 168.2,153.9,152.2,133.6$, 128.8, 126.3, 124.4, 121.9, 121.8, 120.1, 113.7; ESI-MS $m / z: 225\left(\mathrm{M}-\mathrm{H}^{+}\right)$. Anal. calcd for $\mathrm{C}_{13} \mathrm{H}_{10} \mathrm{~N}_{2} \mathrm{~S}: \mathrm{C} 69.03, \mathrm{H}$ 4.42, N 12.39; found C 69.21, H 4.26, N 12.56.

\subsubsection{2-(4-叠氮基苯基)苯并噻唑(FL)的合成}

将中间体 1 ( $0.23 \mathrm{~g}, 1 \mathrm{mmol})$ 溶解在浓盐酸 $(6 \mathrm{~mL})$ 和 乙醇 $(20 \mathrm{~mL})$ 的混合溶液中, 用冰浴冷却反应液到 $0{ }^{\circ} \mathrm{C}$ 后, 再加入亚硝酸钠 $(0.10 \mathrm{~g}, 1.5 \mathrm{mmol})$ 并搅拌 $0.5 \mathrm{~h}$. 然 后将叠氮化钠 $(0.10 \mathrm{~g}, 1.5 \mathrm{mmol})$ 缓慢的加入上述反应液 中, 在室温下又继续摚拌 $2 \mathrm{~h}$. 反应混合物用 $\mathrm{CH}_{2} \mathrm{Cl}_{2}$ 萃 取 $(50 \mathrm{~mL} \times 3)$, 有机层用无水硫酸镁干燥, 旋转蒸发除 去溶剂, 所得粗产品用硅胶柱分离 $[V$ (乙酸乙酯) : $V$ (石 油醚 $)=1: 5$ ] 可得浅黄色的晶状粉末 $0.21 \mathrm{~g}$, 产率 $85 \%$. m.p. $156.5 \sim 158.5{ }^{\circ} \mathrm{C} ;{ }^{1} \mathrm{H}$ NMR $\left(400 \mathrm{MHz}, \mathrm{CDCl}_{3}\right) \delta$ : $8.24(\mathrm{~d}, J=7.8 \mathrm{~Hz}, 2 \mathrm{H}), 7.48 \sim 7.76(\mathrm{~m}, 4 \mathrm{H}), 7.16(\mathrm{~d}, J=$ $4.2 \mathrm{~Hz}, 2 \mathrm{H}) ;{ }^{13} \mathrm{C}$ NMR $\left(\mathrm{CDCl}_{3}, 100 \mathrm{MHz}\right) \delta: 169.8,156.7$, 146.2, 145.6, 135.6, 130.8, 130.4, 128.6, 127.5, 126.2, 123.4, 122.8, 122.3; ESI-MS m/z: $252\left(\mathrm{M}^{+}\right)$. Anal. calcd. for $\mathrm{C}_{13} \mathrm{H}_{8} \mathrm{~N}_{4} \mathrm{~S}$ : C 61.89, H 3.20, N 22.21; found C 61.68, H 3.36, N 22.16 .

\section{3 晶体结构测定及解析}

将大小为 $0.12 \mathrm{~mm} \times 0.10 \mathrm{~mm} \times 0.10 \mathrm{~mm}$ 的浅黄色 方块状晶体 FL 置于 Bruker Smart-2000 CCD 型单晶衍射 仪上, 用石墨单色化的 Mo $\mathrm{K} \alpha$ 射线 $(\lambda=0.071073 \mathrm{~nm})$, 于 $296(2) \mathrm{K}$, 以 $\omega / 2 \theta$ 方式扫描, 在 $1.50^{\circ}<\theta<28.15^{\circ}$ 范 围内共收集到衍射点 17006 个, 其中独立衍射点 2807 个 $\left(R_{\mathrm{int}}=0.0361\right), I>2 \sigma(I)$ 的可观测的衍射点 2274 个, -
$19 \leqslant h \leqslant 17,-7 \leqslant k \leqslant 7,-35 \leqslant l \leqslant 35$. 全部衍射数据经 Lp 因子和经验吸收校正。晶体结构由直接法用 SHELXS-97 程序解出, 部分非氢原子坐标是在以后的 数轮差值 Fourier 合成中陆续确定的. 对全部非氢原子 的坐标及各向异性参数用 SHELXL-97 程序, 以全矩阵 最小二乘法基于 $F^{2}$ 进行精修 $[25,26]$.

\section{4 荧光光谱测量方法}

在乙醇中配制 $1 \mathrm{mmol} \cdot \mathrm{L}^{-1}$ 的探针 $\mathrm{FL}$ 储备液, 用去 离子水配制 $10 \mathrm{mmol} \cdot \mathrm{L}^{-1}$ 的 NaHS 储备液和 $20 \mathrm{mmol} \cdot$ $\mathrm{L}^{-1}$ Tris- $\mathrm{HCl}$ 缓冲液 $(\mathrm{pH}=7.4)$. 各种阴离子 $\left(\mathrm{F}^{-}, \mathrm{Cl}^{-}\right.$, $\mathrm{Br}^{-}, \mathrm{I}^{-}, \mathrm{SCN}^{-}, \mathrm{HCO}_{3}^{-}, \mathrm{CO}_{3}^{2-}, \mathrm{Ac}^{-}, \mathrm{H}_{2} \mathrm{PO}_{4}^{-}, \mathrm{HPO}_{4}^{2-}$, $\mathrm{PO}_{4}^{3-}, \mathrm{SO}_{4}^{2-}, \mathrm{SO}_{3}^{2-}, \mathrm{HSO}_{3}^{-}, \mathrm{HSO}_{4}^{-}, \mathrm{HS}^{-}, \mathrm{NO}_{3}^{-}$, $\mathrm{NO}_{2}^{-}, \mathrm{ClO}^{-}$)检测溶液都采用其钠盐，其储备液的浓度 配制成 $1 \mathrm{mmol} \cdot \mathrm{L}^{-1}, \mathrm{H}_{2} \mathrm{O}_{2}$, 半胱氨酸(Cys), 谷胱甘肽 $(\mathrm{GSH})$ 储备液的浓度配制成 $10 \mathrm{mmol} \cdot \mathrm{L}^{-1}$. 测试苂光光 谱时, 用移液枪取 $10 \mu \mathrm{L} \mathrm{FL}$ 储备液, 加入含有 $1 \mathrm{~mL}$ Tris- $\mathrm{HCl}$ 缓冲体系 $\left[\mathrm{pH}=7.4, V\left(\mathrm{H}_{2} \mathrm{O}\right): V(\mathrm{EtOH})=1: 1\right]$ 的荧光比色血中, 使得测试溶液中 FL 浓度为 $10 \mu \mathrm{mol}$ ・ $\mathrm{L}^{-1}$, 然后加入各种阴离子等检测液, 混合均匀, $5 \mathrm{~min}$ 后测定探针的荧光光谱. 测试所用的激发波长为 325 $\mathrm{nm}$, 狭缝宽度为 $10 \mathrm{~nm} / 10 \mathrm{~nm}$.

\section{5 各种水体样品中硫化氢的检测}

参照文献的方法进行各种水体样品硫化氢检测的 恢复试验 ${ }^{[27]}$. 实验中选择的三种水体样本分别为自来 水、湖水和工业废水, 其中自来水取自化学实验室, 湖 水来自于湖北黄石市磁湖水样, 工业废水为湖北黄石市 某有色金属冶炼厂产生的废水. 自来水和湖水没有作任 何处理进行实验测试，工业废水先过滤，取滤液用于实 验. 测试时, 以各种水样代替荧光测试所用的蒸馏水, 其它和上述荧光光谱测量方法一样.

辅助材料(Sopporting Information) 细胞造影和毒性 实验的方法和图片, 以及向 FL 中加入 $\mathrm{NaHS}$ 后反应液 的质谱图. 这些材料可以免费从本刊网站(http://siocjournal.cn/)上下载.

\section{References}

[1] Li, L.; Rose, P.; Moore, P. K. Annu. Rev. Pharmacol. Toxicol. 2011, 51, 169.

[2] Kabil, O.; Banerjee, R. J. Biol. Chem. 2010, 285, 21903.

[3] Lefer, D. J. Proc. Natl. Acad. Sci. U. S. A. 2007, 104, 17907.

[4] Blackstone, E.; Morrison, M.; Roth, M. B. Science 2005, 308, 587.

[5] Huang, B.; Chen, Y.-C.; Guo, Z.-J.; He, W.-J. Chin. J. Inorg. Chem. 2013, 29, 2283 (in Chinese).

(黄涁, 陈韵聪, 郭子建, 何卫江, 无机化学学报, 2013, 29, 2283.)

[6] Liu, J.; Sun, Y.; Zhang, J.; Yang, T.; Cao, J.; Zhang, L.; Guo, W. Chem. Eur. J. 2013, 19, 4717. 
[7] Kumar, N.; Bhalla, V.; Kumar, M. Coord. Chem. Rev. 2013, 257, 2335.

[8] Jiang, Y.; Wu, Q.; Chang, X. Talanta 2014, 121, 122.

[9] Zhang, J.; Guo, W. Chem. Commun. 2014, 50, 4214.

[10] Mao, G.; Wei, T.; Wang, X.; Huan, S.; Lu, D.; Zhang, J.; Zhang, X.; Tan, W.; Shen, G.; Yu, R. Anal. Chem. 2013, 85, 7875.

[11] Chen Y.; Zhu, C.; Yang, Z.; Chen, J.; He, Y.; Jiao, Y.; He, W.; Qiu, L.; Cen, J.; Guo, Z. Angew. Chem., Int. Ed. 2013, 52, 1688.

[12] Wang, X.; Sun, J.; Zhang, W.; Ma, X.; Lv, J.; Tang, B. Chem. Sci. 2013, 4, 2551.

[13] Lippert, A. R.; New, E. J.; Chang, C. J. J. Am. Chem. Soc. 2011, 133, 10078.

[14] Peng, H.; Cheng, Y.; Dai, C.; King, A. L.; Predmore, B. L.; Lefer, D. J.; Wang, B. Angew Chem., Int. Ed. 2011, 50, 9672.

[15] Montoya, L. A.; Pluth, M. D. Chem. Commun. 2012, 48, 4767.

[16] Zhou, G.; Wang, H.; Ma, Y.; Chen, X. Tetrahedron 2013, 69, 867.

[17] Li, W.; Sun, W.; Yu, X.; Du, L.; Li M. J. Fluoresc. 2013, 23, 181.
[18] Wan, Q.; Song, Y.; Li, Z.; Gao, X.; Ma, H. Chem. Commun. 2013, 49, 502.

[19] Hartman, M. C. T.; Dcona. M. M. Analyst 2012, 137, 4910.

[20] Yu, F.; Li, P.; Song, P.; Wang, B.; Zhao, J.; Han, K. Chem. Commun. 2012, 48, 2852.

[21] Huang, Q.; Yang, X.; Li, H. Dyes Pigm. 2013, 99, 871.

[22] Yang, Y.; Li, B.; Zhang, L.; Guan, Y. J. Lumin. 2014, 145, 895.

[23] Shortreed M, Kopelman R, Kuhn M, Hoyland, B. Anal. Chem. 1996, 68, 1414.

[24] Shi, D. F.; Bradshaw, T. D.; Wrigley, S.; McCall, C. J.; Lelieveld, P.; Fichtner, I.; Stevens, M. F. G. J. Med. Chem. 1996, 39, 3375.

[25] Sheldrick, G. M. SHELXS-97, Program for the Solution of Crystal Structures, University of Göttingen, Germany, 1997.

[26] Sheldrick, G. M. SHELXL-97, Program for the Refinement of Crystal Structures, University of Göttingen, Germany, 1997.

[27] Liu, Y.; Fei, Q.; Shan, H.; Cui, M.; Liu, Q.; Feng, G.; Huan, Y. Analyst 2014, 139, 1868. 


\title{
辅助材料(Supporting Information)
}

\section{一种可视化检测硫化氢的苯并噻唑类荧光增强型探针}

\author{
范方禄* 靖金球陈雪梅 \\ (湖北理工学院化学与材料工程学院 黄石 435003)
}

\section{1 苂光量子产率的测定}

测试时, 以硫酸奎宁为参比荧光标准物质(在 $0.1 \mathrm{~mol} \cdot \mathrm{L}^{-1}$ 的硫酸中其荧光量子产率为 0.55 ). 首先配制 $10 \mu \mathrm{mol} \cdot \mathrm{L}^{-1}$ FL 和中间体 1 的乙醇溶液, 调整与它们有相近紫外吸收的硫酸奎宁的浓度, 使两者的吸光度相近且不大于 0.05 , 然后测定 FL、中间体 1 和硫酸奎宁在 325-550 nm 范围内的积分苂光强度, 结果取三次平均值, 最后按下式计算苂 光量子产率:

$$
Y_{\mathrm{u}}=Y_{\mathrm{s}} \cdot \frac{F u}{F s} \cdot \frac{A s}{A u}
$$

$Y_{\mathrm{u}}, Y_{\mathrm{s}}$ 一待测物质和参比标准物质的苂光量子产率;

$F_{\mathrm{u}}, F_{\mathrm{s}}$ 一为待测物质和参比物质的积分荧光强度;

$A_{\mathrm{u}}, A_{\mathrm{s}}$ 一为待测物质和参比物质在该激发波长的入射光的吸光度.

\section{2 细胞造影实验}

将事先培养好的 HeLa 细胞首先用磷酸盐缓冲溶液(PBS)清洗 3 次, 然后在 Tris- $\mathrm{HCl}$ 缓冲体系中加入 $5 \mu \mathrm{mol} \cdot \mathrm{L}^{-1} \mathrm{FL}$ 溶液 $\left(\mathrm{pH}=7.4, \mathrm{H}_{2} \mathrm{O}: \mathrm{EtOH}=1: 1, \mathrm{~V} / \mathrm{V}\right)$, 在 $37{ }^{\circ} \mathrm{C}$ 恒温培养 $30 \mathrm{~min}$, 再用 $\mathrm{PBS}$ 缓冲液洗 3 次以便除去探针溶液, 将其放 在倒置荧光显微镜下, 用绿光激发来观察细胞的苂光成像. 再向上述含 FL 的细胞培养液中加入 NaHS 溶液(100 $\mu \mathrm{mol} \cdot \mathrm{L}^{-1}$ ), 在 $37{ }^{\circ} \mathrm{C}$ 继续恒温捊育 $30 \mathrm{~min}$, 然后用 $\mathrm{PBS}$ 缓冲液清洗 3 次, 观察 $\mathrm{FL}$ 在细胞中对 $\mathrm{H}_{2} \mathrm{~S}$ 的造影. 相对于细 胞内的 FL 苂光强度, 对细胞的明亮视野也进行了成像, 放大倍数为 400 倍.

\section{3 细胞毒性实验}

探针对细胞的毒性实验采用 MTT 法进行测定. 将 HeLa 细胞种植于 96 孔板中, 保证细胞密度为 $1 \times 10^{5} \mathrm{cells} / \mathrm{mL}$. 细 胞培养 $24 \mathrm{~h}$ 后, 加入 $5 \mu \mathrm{mol} \cdot \mathrm{L}^{-1}$ 的 FL 或中间体 1, 分别培养细胞 $12 \mathrm{~h}$ 和 $24 \mathrm{~h}$, 不加探针的细胞也分别培养 $12 \mathrm{~h}$ 和 $24 \mathrm{~h}$ 作为对照组. 然后每孔加入 $20 \mu \mathrm{L}$ MTT 溶液 $(5 \mathrm{mg} / \mathrm{mL}$, 即 $0.5 \% \mathrm{MTT}$ ), 继续培养 $4 \mathrm{~h}$. 终止培养, 小心吸 去孔内培养液. 每孔加入 $150 \mu \mathrm{L}$ DMSO, 置摇床上低速振荡 $10 \mathrm{~min}$. 最后通过在酶标仪 OD $490 \mathrm{~nm}$ 处测量 各孔的吸光值, 评价探针的细胞毒性. 


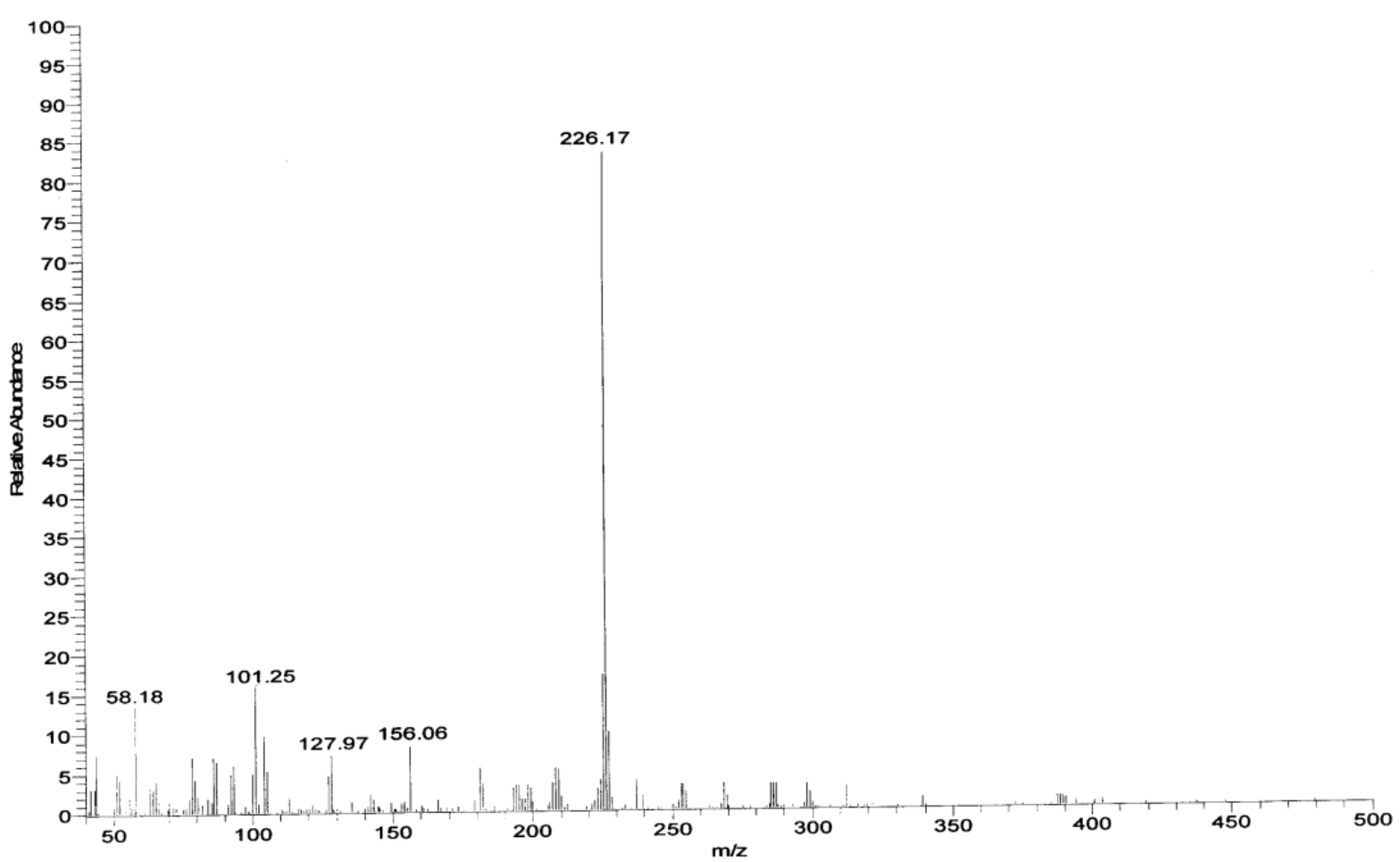

图 S1 向探针 FL 中加入 $\mathrm{NaHS}$ 后反应液的质谱

Figure S1 MS chart of the solution of probe FL upon addition of NaHS

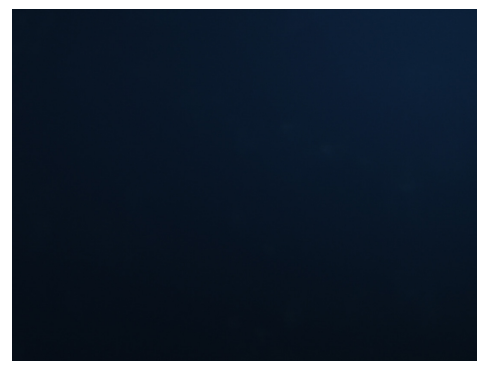

(a)

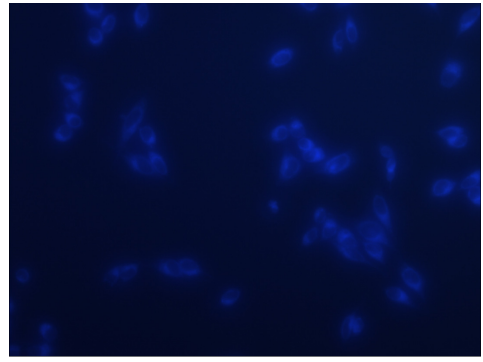

(b)

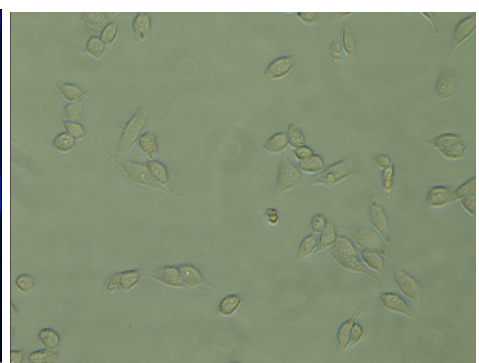

(c)

图 S2 探针 $\mathrm{FL}$ 在 $\mathrm{HeLa}$ 细胞中对 $\mathrm{H}_{2} \mathrm{~S}$ 的造影.(a) $\mathrm{HeLa}$ 细胞与 $5 \mu \mathrm{mol} \cdot \mathrm{L}^{-1} \mathrm{FL}$ 于 $37^{\circ} \mathrm{C}$ 共培养 $30 \mathrm{~min}$; (b) 在 $37^{\circ} \mathrm{C}$ 的条件下再 向(a)中的 HeLa 细胞加入 $100 \mu \mathrm{mol} \cdot \mathrm{L}^{-1} \mathrm{NaHS}$ 并孵化 $30 \mathrm{~min}$; (c) (b)中 HeLa 细胞的亮区影像

Figure S2 Fluorescence images of $\mathrm{H}_{2} \mathrm{~S}$ in HeLa cells using probe FL. (a) HeLa cells incubated with $5 \mu \mathrm{mol} \cdot \mathrm{L}^{-1} \mathrm{FL}$ for $30 \mathrm{~min}$ at $37{ }^{\circ} \mathrm{C}$; (b) HeLa cells from (a) after treatment with $100 \mu \mathrm{mol} \cdot \mathrm{L}^{-1} \mathrm{NaHS}$ for $30 \mathrm{~min}$ at $37^{\circ} \mathrm{C}$; (c) the bright-field images of HeLa cells from (b) 


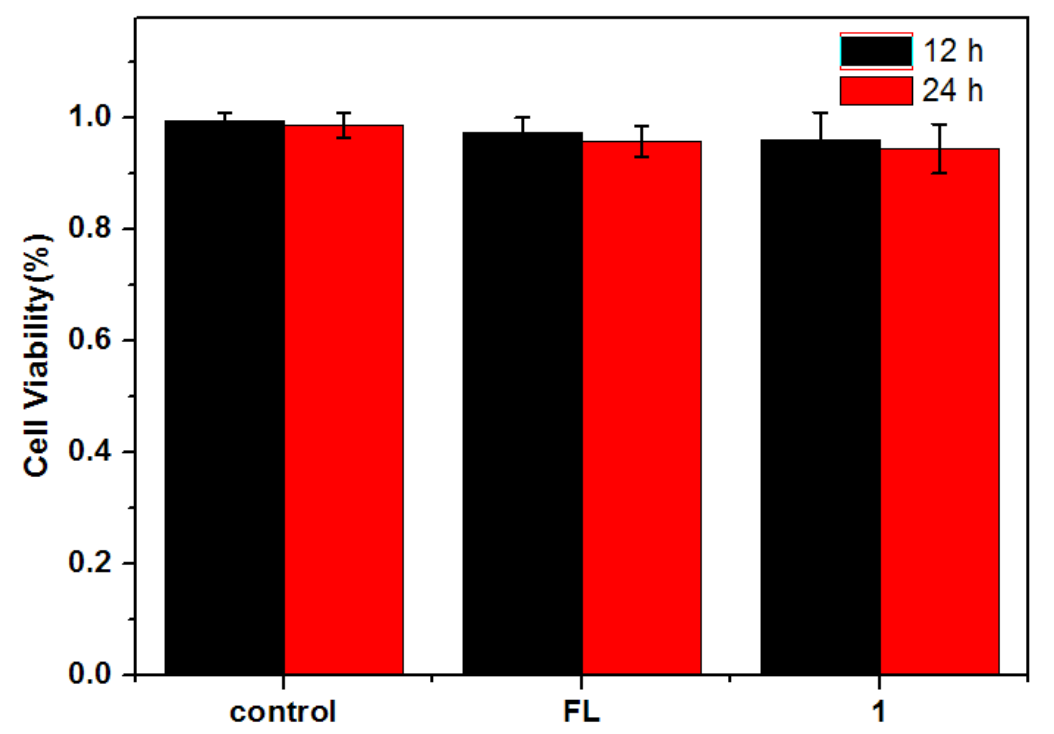

图 S3 探针 FL 和中间体 $1\left(5 \mu \mathrm{mol} \cdot \mathrm{L}^{-1}\right)$ 在 $\mathrm{HeLa}$ 活细胞中培养 $12 \mathrm{~h}$ 和 $24 \mathrm{~h}$ 的毒性

Figure S3 Cytotoxicity of probe FL and intermediate $1\left(5 \mu \mathrm{mol} \cdot \mathrm{L}^{-1}\right)$ in living HeLa cells for $12 \mathrm{~h}$ and $24 \mathrm{~h}$ 